\title{
ARTICLE
}

\section{A Study on Beam Flattening Based on Compact Double Scatterer Applicable to Rotational Beam Irradiation System in the Proton Therapy Facility at CYRIC, Tohoku University}

\author{
Ahmad ESMAILI TORSHABI ${ }^{1, *}$, Atsuki TERAKAWA ${ }^{2}$, Keizo ISHII $^{2}$, Hiromichi YAMAZAKI ${ }^{3}$, Shigeo MATSUYAMA ${ }^{2}$, \\ Youhei KIKUCHI ${ }^{2}$, Hisaki AKIYAMA ${ }^{2}$, Kan KOYATA ${ }^{2}$, Atsushi TAGAWA ${ }^{2}$, Yuki ITOH ${ }^{2}$, Shigeyoshi YASUNAGA ${ }^{2}$ \\ ${ }^{1}$ Centro Nazionale di Adroterapia Oncologia CNAO, Strada Privata Campeggi, Pavia 27100, Italy \\ ${ }^{2}$ Department of Quantum Science and Energy Engineering, Graduate School of Engineering, Tohoku University, Aoba-ku, Aramaki \\ Aza-Aoba 6-6-01-2, Sendai 980-8579, Japan \\ ${ }^{3}$ Cyclotron and Radioisotope Center, Tohoku University, Aoba-ku, Aramaki Aza-Aoba 6-3, Sendai 980-8579, Japan
}

\begin{abstract}
Proton therapy facility at Cyclotron and Radioisotope Center (CYRIC) in Tohoku University includes a horizontal beam line, currently used for research studies, and also a rotational beam irradiation system which is under construction. This system will be able to change the beam irradiation angle from 0 to 180 degree around the target and the beam delivery on the target volume is done passively using scatterers and ridge filters. In this rotational beam irradiation system due to the limitation on the available space between the beam exit window to the target location, a compact compensated contoured double scattering system was proposed to create the lateral homogeneous dose profile. This work represents the evaluation of this compact double scatterer and its application to the rotational beam irradiation system. The experiments were performed using the horizontal beam line by considering to the status of the rotational beam irradiation system in the target room 5 at CYRIC. The experimental results represent the lateral dose distribution with a proper treatment region which is in a good agreement with simulated data.
\end{abstract}

KEYWORDS: compact compensated contoured double scatterer, rotating gantry system, proton therapy

\section{Introduction}

In proton therapy, two major beam delivery techniques are used, which are referenced as active and passive beam delivery techniques ${ }^{1,2,12)}$. Active beam delivery uses a pencil beam which is scanned magnetically over the tumor cross section $^{3}$. In the passive beam delivery technique an effective treatment beam is created by spreading pencil beam extracted from the accelerator to produce two uniform dose profiles; laterally and in depth, known as Spread-Out Bragg Peak (SOBP). To achieve SOBP, several types of ridge filters are employed in front of proton beam to give desired homogeneous dose in depth ${ }^{4}$. In lateral direction, broad and uniform beam profile is produced using double scattering technique ${ }^{5-7)}$ or single scattering in combination with beam wobbling system ${ }^{8)}$. The wobbling system includes two separate magnets in series for horizontal and vertical deflecting of protons along their central axis in a circular fashion.

In double scattering system the flat beam profile is created by a simple and uniform foil as first scatterer in combination with a non-uniform scattering foil as second scatterer. This system can be made in three basic methods which are occluding ring ${ }^{6}$, dual ring double scattering ${ }^{7)}$ and compensated contoured double scattering methods.

\footnotetext{
* Corresponding Author, Tel. No.: +39382078510,

Fax No.: +39382079926,

E-mail: torshabi@cnao.it

Present address: Fondazione CNAO, Strada Privata Campeggi,

Pavia 27100, Italy
}

Proton therapy facility of Cyclotron and Radioisotope Center (CYRIC) in Tohoku University includes a horizontal beam line which is currently used for research experiments and a rotating gantry system, which is under construction ${ }^{9,11 \text {, }}$ 13). Horizontal beam line includes beam wobbling system with single scatterer to produce lateral homogeneous dose but in the rotating gantry, as the distance between beam exit window to target location is short (around $500 \mathrm{~mm}$ ) and the installation of the wobbling system is impossible, beam spreading can be done by double scattering technique. For this aim, a compact compensated contoured double scattering system was constructed to produce a lateral homogeneous dose with treatment area around $60 \mathrm{~mm}$ which is suitable for dose delivery on targets volume in this research center. Since rotating gantry is under construction, experimental tests were done by horizontal beam line with consideration to rotating gantry status.

\section{Material and method}

\section{Proton therapy facility at CYRIC}

Proton therapy facility at CYRIC includes a horizontal beamline for research experiments and consists of beam wobbling system, beam monitoring system, different types of ridge filter, range shifter, collimators, detectors and other required devices for doing experiment ${ }^{11}$. Current works are study on therapeutic effects of protons on in-vivo tumor cells, tumor irradiation using mouse, development of dose delivery system, beam monitoring system and work on SOBP. In this center protons are accelerated by AVF (Azimuthally Varying Field) cyclotron and their energy reach to $80 \mathrm{MeV}$. A 
rotating gantry system employing beam swinger is under construction which is able to change beam irradiation angle from 0 to 180 degree. Figure 1 shows a schematic layout of this system.

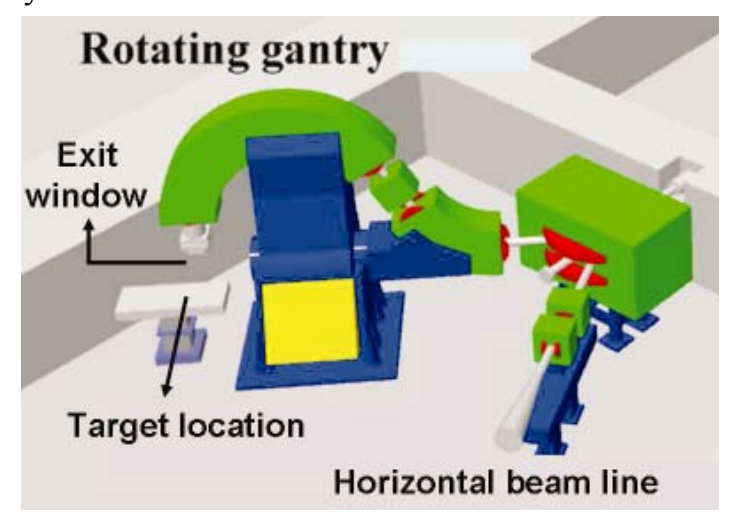

Fig. 1 Schematic layout of horizontal and rotating gantry systems in the Proton therapy facility at CYRIC

\section{Flat beam generation}

While irradiation using horizontal beam line, lateral homogeneous dose is generated by single scatterer with wobbling system but in the rotating gantry as the distance between beam exit window to target location is not long enough for wobbling system installation; a compact compensated contoured double scatterer was constructed to produce a flat beam profile with reasonable treatment area. Figure 2 shows a schematic layout of contoured double scatterer and its operation in spreading protons.

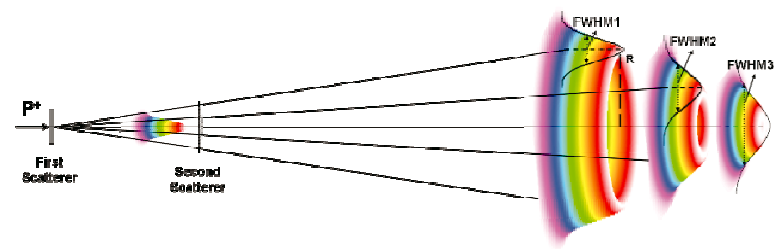

Fig. 2 Schematic layout of three dose distribution profiles created by scattered protons emerged from three circular sections on the second scatterer (as samples).

Protons leaving the first scatterer are spread in a range of angles as a net result of interactions in their paths due to the process of multiple coulomb scattering ${ }^{10)}$. The distribution of these angles is very close to Gaussian distribution with a specific FWHM (Full Width at Half Maximum). Thus, the narrow pencil beam will be expanded into a larger beam until it reaches to the second scatterer. In the second scatterer, since it has cone shape, the scattering strength is decreased from its center to outer parts. Figure 2 illustrates the contribution of protons emerged from three circular sections on the second scatterer to generate dose distributions. The profiles of these distributions have been shown separately in series, for better description. As shown in this Fig. , the first 3D donut shaped dose distribution downstream of the scatterers specified by FWHM1, is created by the thinnest circular section on the second scatterer and the third 3D dose distribution specified by FWHM3 (>FWHM2 $>$ FWHM1) is created by the thickest part, which is in the center. Although Fig. 2 shows just three dose profiles as samples, but by adding all dose profiles resulted by each section of the second scatterer, the flat beam profile is generated including a circular treatment area with radius $\mathrm{R}^{14)}$.

From computational point of view, the fluence at each point on measuring plate depends on a scatter event through the first scatterer to a point on the second scatterer and then another scatter event from that point on the second scatterer to a point on the measuring plate, integrated for all points on second scatterer. In this work Monte Carlo MCNPX code was used for simulating the physical system and evaluating the flat lateral dose distributions. The Monte Carlo method is a well-established technique that has found widespread uses in different situations. In the context of radiation transport, the technique involves the use of random numbers and probability distributions to determine the energy, position, direction and path-length of individual particles, as well as the type of physical interactions that particles experience when traversing a medium.

In this work, the geometry of contoured double scattering system (scatterers thickness, distances, etc.) were simulated by Monte Carlo MCNPX (v26c) code and in a trial and error method the best thickness of the first scatterer and the best shape of the second scatterer (thickness of the cone at each point) were obtained in order to give the proper flat dose profile.

As the second scatterer has cone shape, the energy loss of protons emerged from different points on the second scatterer is not uniform and this inconsistency can be compensated by a proper compensator. The compensator causes constant energy loss for protons leaving the second scatterer. Figure 3 represents the combination of the second scatterer with compensator. As shown, the thickness of compensator is variable according to the shape of the second scatterer.

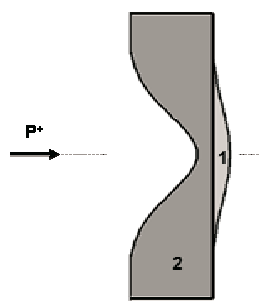

Fig. 3 Schematic layout of the second scatterer (number 1) with compensator (number 2)

In this work Lead was used as the first and second scatterers material, because of its high density $\left(11.34 \mathrm{gr} / \mathrm{cm}^{3}\right)$ and Polymethyl methacrylate (PMMA) was used as compensator material because of its low density (1.19 $\mathrm{gr} / \mathrm{cm}^{3}$ ) to attenuate the protons without spreading them. The thickness of the first scatterer and thickest part of the second scatterer are $1.25 \mathrm{~mm}$ and $1.33 \mathrm{~mm}$, respectively. The distance between the first and second scatterers of this system is $100 \mathrm{~mm}$ which can save enough space for using 
other devices such as ridge filter or beam monitoring system. The location of the scattering system was very close to beam exit window on horizontal beam line. Imaging Plate $^{1}$ was employed to measure beam profiles and was located at $500 \mathrm{~mm}$ distance from the first scatterer. Although the horizontal beam line was used while irradiation but the status of rotating gantry system was considered in this work.

\section{Results}

Figure 4 represents the measured and simulated data of the lateral flat beam profile created with $80 \mathrm{MeV}$ proton beam. The given diameter of treatment area was $60 \mathrm{~mm}$ while designing scatterer system (treatment area 1) and the treatment area of the measured flat dose profile has $57 \mathrm{~mm}$ diameter (treatment area 2). The treatment areas 1 and 2 have been determined according to flatness definition and the value of flatness is $\pm 2.5 \%$ in this work. The differences between simulated and measured data comes from experimental errors such as beam energy fluctuation which is $\Delta \mathrm{E}= \pm 0.5 \mathrm{MeV}$, scatterers material impurity and beam imperfection. In simulation process initial pencil beam has a perfect Gaussian shape, but in experimental situation the shape of the initial beam is near Gaussian which affects of final flat beam profile.

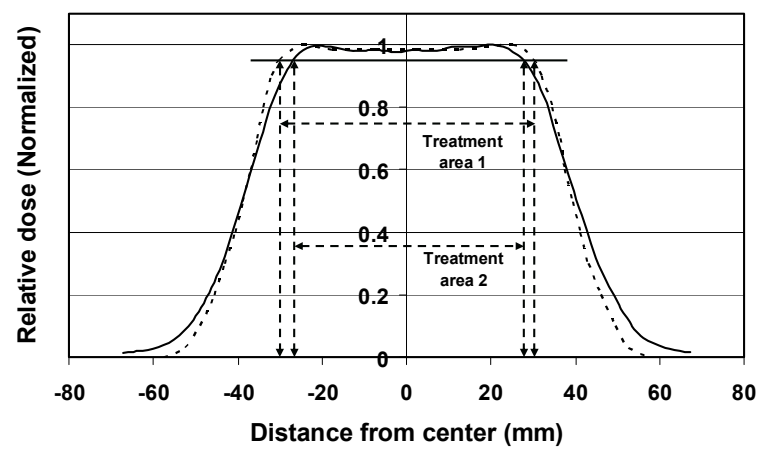

Fig. 4 Flat beam profiles of measured (Solid line) and simulated methods (Dashed line)

Figure 5 shows the Bragg peak profiles of the predicted and measured data inside water. There is $1 \mathrm{~mm}$ difference between simulated and measured data in distal part of depth dose profiles which is resulted from experimental errors as mentioned above and also the presence of air atomic compositions between scatterer system and IP. Since the thickness of the first and second scatterers of our scattering system is not negligible, the energy loss is around $5 \mathrm{MeV}$ in this system.

\footnotetext{
${ }^{1}$ Imaging Plate (IP) made by Fuji Photo film CO. Ltd
}

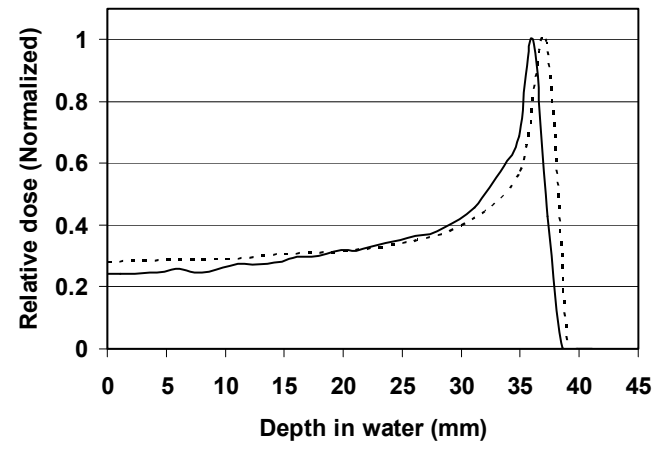

Fig. 5 Depth dose profiles of measured (Solid line) and simulated (Dashed line) data

It should be considered that the contoured double scatterer is very sensitive in aligning with beam direction and this is the main disadvantage of the contoured scattering system in comparison with single scattering with beam wobbling system for flat beam generation. Figure $\mathbf{6}$ shows the effect of the slightly misaligned (less than $1 \mathrm{~mm}$ off-center) scattering system on the beam profile generation in comparison with flat beam profile created with aligned scattering system.

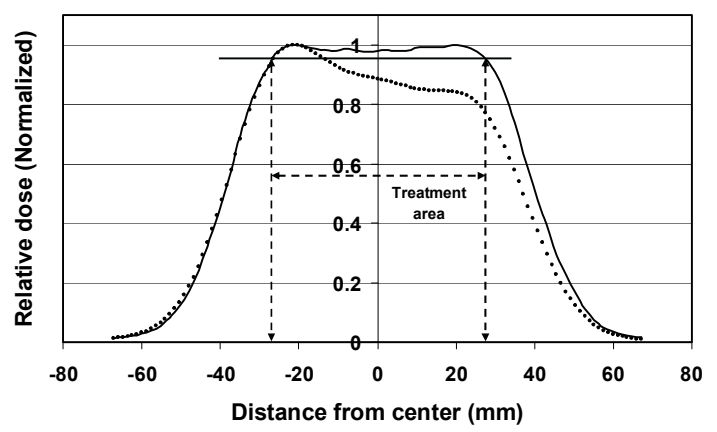

Fig. 6 Tilted (Dotted line) and flat beam profiles (Solid line) produced by misaligned and aligned double scattering system

\section{Conclusion}

A rotating gantry system is under construction in proton therapy facility at CYRIC and there is a need to spread the exited pencil beam from this system into a lateral expanded beam with a flat treatment area. In the rotating gantry system, since the distance between beam exit window to target location is short, a compact compensated contoured double scatterer was constructed to produce a reasonable lateral homogeneous dose and the experimental tests was done using horizontal beam line which is a separate irradiation line near rotating gantry system.

The diameter of the treatment area created by our system is $57 \mathrm{~mm}$ which is suitable for research works in this center. This system was simulated with Monte Carlo MCNPX code which gave the best thickness size of both scatterers and represents the lateral and depth dose profiles according to the beam characters. 
The sensitivity of this system to its alignment with beam direction is a main disadvantage which must be considered while applying this system.

\section{References}

1) A. Smith, "Proton therapy," Phys. Med. Biol., 51, 491-504 (2006).

2) J. R. Castro, P. T. Petti, A.K. Daftari, et al., "Clinical gain from improved beam delivery systems," Radiat. Environ. Biophys., 31, 233-240 (1992).

3) A. Lomax, "Intensity modulation methods for proton radiotherapy," Phys. Med. Biol., 44, 185-205 (1999).

4) T. Akagi, A. Higashi, H. Tsugami, et al., "Ridge filter design for proton therapy at Hyogo Ion Beam Medical Center," Phys. Med. Biol., 48, 301-312 (2003).

5) E. Grusell, A. Montelius, A. Brahme, et al., "A general solution to charged particle beam flattening using an optimized dual-scattering-foil technique, with application to proton therapy beams," Phys. Med. Biol., 39, 2201-2216 (1994).

6) A. M. Koehler, R. J. Schneider, J. M. sisterson, "Flattening of proton dose distributions for large-field radiotherapy," Med. Phys., 4[4], 297-301 (1977).

7) Y. Takada, "Dual-ring double scattering method for proton beam spreading,” Japan J. Appl. Phys., 33, 353-359 (1994).

8) Timothy R. Renner, and William T. Chu, "Wobbler facility for biomedical experiments," Med. Phys., 14, 824-834 (1987).

9) A. Terakawa, H. Suzuki, K. Kumagai, et al., "New fast-neutron time-of-flight facilities at CYRIC," Nucl. Inst. and meth. in Phys. Res. A, 491, 419-425 (2002).

10) B. Gottschalk, A. M. Koehler, R. J. Schneider, et al., "Multiple coulomb scattering of $160 \mathrm{MeV}$ protons," Nucl. Inst. and Meth. in Phys. Res. B, 74, 467-490 (1993).

11) A. Terakawa, A. Ishizaki, Y. Totsuka, et al., "Beam Irradiation System for Proton Therapy at CYRIC," CYRIC annual report, 41-43 (2005).

12) E. Pedroni, "LATEST DEVELOPMENTS IN PROTON THERAPY," Proc. of the $7^{\text {th }}$ European Particle Accelerator Conference (EPAC), Vienna, Austria, June, 2000, 240- 244 (2000). 13) A. Terakawa, K. Ishii, T. Chiba, et al., "Proton Therapy facilities at CYRIC, Tohoku University," Proc. of the $16^{\text {th }}$ Pacific Basin nuclear conference (16PBC), Aomori, Japan, Oct., 13-18, 2008, 1-6 (2008).

14) E. W. Cascio, "The Use of the Compensated Contoured Double scatterer in the Radiation test beamline at the Francis H. Burr Proton Therapy Center," Radiation Effects Data Workshop, IEEE, 2006, 177-182 (2006). 